Contribuciones originales sobre el complejo mundo

de la educación química.

\title{
Enseñanza/aprendizaje de la ciencia versus historia de la ciencia
}

\author{
Joan Josep Solaz-Portolés y Magdalena Moreno-Cabo ${ }^{2}$
}

\section{Abstract (Teaching/learning of science versus history of science)}

Our discussion will focus on how the history of science can give science teachers some insight about students' alternative ideas, and provides some teaching materials and approaches which can be used in classroom in order to change students' ideas. Besides, we analyze the differences between the progression of scientific understanding in the pupil and the progression of scientific understanding in the history of science.

\section{Introducción}

Podemos encontrar en la literatura abundantes argumentos a favor de la introducción de la historia de la ciencia en el curriculum de las asignaturas científicas de la enseñanza secundaria (Dana, 1990; Solomon, 1992). En este sentido, Nielsen y Thomsen (1990) señalan como beneficios obtenidos por los estudiantes que cursan asignaturas impregnadas de historia de la ciencia los siguientes:

- Comprender el modo de desarrollo de la ciencia

- Generar actitudes positivas hacia la ciencia

- Entender la relevancia cultural/social de la ciencia

- Mejorar la asimilación de los conceptos científicos.

Por otro lado, la situación educativa real, que se plasma en los libros de texto, refleja un panorama bastante diferente. Así, podemos referirnos aquí a ciertos trabajos que han puesto el acento en las deficiencias histórico-epistemológicas que muestran los textos para la enseñanza de las ciencias fisicoquímicas. Strube (1989), en un análisis de argumentos y explicaciones presentados en textos de Física General y Química General (preuniversitarios y universitarios) ingleses y australianos, revela la ausencia de pasajes que discutan la función de teorías, leyes y modelos en los procesos de indagación científica. Chiappetta y colaboradores (1991) examinan el contenido de textos de Química de High School, y obtienen que presentan un cuerpo de conocimientos elaborados de manera acumulativa, sin ningún tipo de error, y además no prestan importancia alguna al desarrollo

1 I.B. Camp de Túria, 46160 Llíria, València / C.A. "F. Tomás y Valiente" de la UNED, España.

2 B.P.M. de L'Eliana, València, España.

*Recibido: 11 de julio de 1997; Aceptado: 29 de octubre de 1997. histórico de los conceptos. Solaz-Portolés y colaboradores (1993) encuentran que en el tema de "Modelos atómicos" de los libros de Física y Química del bachillerato del estado español los autores no dan explicaciones acerca del papel que desempeñan los modelos en la construcción de la ciencia, ni justifican adecuadamente la sustitución de un modelo por otro atendiendo a las dificultades que uno presentó y el otro obvió. Finalmente, tanto el canadiense Stinner (1992) como el norteamericano Brackenbridge (1991) coinciden en afirmar que la imagen de la ciencia que proporcionan los libros de texto es distorsionada, y es fruto del vacío histórico y filosófico que muestran sus páginas.

En el presente trabajo pretendemos poner de relieve la importancia que tiene el conocimiento de la historia de la ciencia para el profesor de ciencias. Como veremos, esta última aseveración se fundamenta en que la historia de la ciencia posibilita, por una parte, anticipar las dificultades conceptuales y modos de razonamiento de nuestros alumnos, y por otra, diseñar actividades que permiten sacar a la luz los esquemas conceptuales de los estudiantes y trabajar sobre ellos.

\section{Ideas previas en los alumnos}

Autores de diferentes escuelas psicológicas han señalado la particular importancia de las concepciones previas de los estudiantes en el aprendizaje. $\mathrm{Al}$ respecto, podemos citar a los autores de la perspectiva cognitiva (Ausubel, 1986), de la perspectiva del desarrollo (Piaget, 1979), de la perspectiva conductual (Gagné, 1987) o la perspectiva constructivista (Driver, 1986). De hecho, diversos estudios en el ámbito de la psicología cognitiva tomaron en consideración el conocimiento previo del aprendiz para la formulación de la teoría de los esquemas como estructuras donde se almacena el conocimiento (Rumelhart, 1980; Shank, 1977).

En los años recientes, la literatura de educación científica está repleta de trabajos relacionados con la identificación, explicación y mejora de las dificultades de los estudiantes en la comprensión de conceptos científicos. Tales dificultades han sido denominadas de diferentes modos: concepciones erróneas o errores conceptuales (misconceptions), esquemas alternativos (alternative frameworks), creencias intuitivas (intuitive beliefs), preconcepciones (preconceptions), razonamiento espontáneo (spontaneous reasoning), ciencia de los niños (children's science), creencias ingenuas (naive beliefs), concepciones alternativas (alternative conceptions), conocimiento del sentido común (common sense knowledge), ciencia 
de los alumnos (pupils'science), concepciones de los estudiantes (students' conceptions) o representaciones de los alumnos (pupils' representations).

Los educadores en el área de las ciencias son sabedores bien recientemente de la importancia de las preconcepciones en la mejora de la enseñanza/aprendizaje de las ciencias. Los estudios de los errores conceptuales de los estudiantes cubren un amplio abanico de conceptos y áreas de conocimiento: fuerza, luz, energía, electricidad, fotosíntesis, cadenas alimenticias, sistema circulatorio, reacciones químicas, equilibrio químico, mol, naturaleza corpuscular de la materia, etcêtera. Las ideas de los estudiantes en diferentes áreas han sido recogidas por diferentes investigadores (West y Pines, 1985; Hierrezuelo y Montero, 1989; Driver, Guesne y Tiberghien, 1989; Osborne y Freyberg, 1991). De sus trabajos podemos concluir que:

1. Los estudiantes tienen ideas y puntos de vista en muchos temas de las ciencias, incluso desde los primeros años de su vida y antes de recibir cualquier tipo de educación formal sobre el tema.

2. Estas descripciones ingenuas y preconcepciones explicativas difieren normalmente de las que mantienen los científicos, pero resultan útiles y coherentes para los estudiantes.

3. Son comunes en estudiantes de diferentes países.

4. A pesar de que los preconceptos tienen una influencia decisiva en el aprendizaje que se lleva a cabo en el aula, los profesores suelen desconocerlos.

5. Son resistentes al cambio por métodos de instrucción tradicional. Esto es, los esquemas alternativos suelen verse inalterados por la instrucción recibida, si ésta no los tiene explícitamente en consideración.

6. Guardan cierto paralelismo con concepciones vigentes en ciertos momentos de la historia de la ciencia (este punto será tratado con detalle más tarde).

Puede encontrarse en la bibliografía un amplia cantidad de hipótesis acerca de las causas de las concepciones alternativas de los alumnos. En este sentido, se apunta la gran influencia de las experiencias físicas y sensoriales de los estudiantes (Driver, 1989), de la interacción con los profesores (Gilbert, 1985), de la sociedad (Solomon, 1987), del lenguaje (Llorens, 1989), de los libros de texto (Andersson 1990; Solaz-Portolès, 1996) o de una metodología de enseñanza/aprendizaje de las ciencias inadecuada (Gil, 1993).

Todo este conjunto de causas puede, según Pozo y colaboradores (1991), clasificarse en tres grandes grupos atendiendo a sus posibles orígenes:

- Origen sensorial, cuando se basan esencialmente en el uso de reglas de inferencia causal aplicadas a los datos procedentes del mundo natural.

- Origen social, cuando reflejan las concepciones inducidas en el alumno por impregnación de las creencias compartidas por su entorno social.

- Origen analógico, cuando proceden de la activación de esquemas de conocimiento inapropiados buscando similitudes superficiales para poder explicar situaciones que los alumnos desconocen.

\section{Aprendizaje de la ciencia, epistemología e historia de la ciencia}

El psicólogo Jean Piaget reforzó las conexiones entre epistemologia y aprendizaje científico, ya sugeridas previamente bien por científicos o bien por epistemólogos. En su Introducción a la Epistemología Genética (Piaget, 1974) el autor ya señalaba que la historia de la ciencia proporcionaba sugerencias sobre cómo conducir e interpretar la investigación en psicología genética, y a su vez, esta investigación daba criterios para seleccionar, organizar y reinterpretar el trabajo de los historiadores de la ciencia. Piaget formuló su hipótesis fundamental del paralelismo entre el desarrollo cognitivo individual y el desarrollo histórico en Epistemología Genética (Piaget, 1970). Esta hipótesis ha sido interpretada por algunos autores (como por ejemplo, Matthews, 1991, p. 147), como "en el desarrollo cognitivo la ontogenia recapitula la filogenia" y frecuentemente ha sido mal interpretada. Sin embargo, es con la publicación de Psicogénesis e historia de la ciencia (Piaget, 1982) donde se presentan los más extensos argumentos en relación con las características comunes del desarrollo individual e histórico, esto es, se plantea un modelo para las relaciones entre el desarrollo individual e histórico. El objetivo del trabajo de Piaget y Garcia es estudiar los instrumentos que conducen al crecimiento y desarrollo del conocimiento en ambos dominios, psicogenético e histórico. En él se compara la psicología genética piagetiana con la historia de la mecánica, de la geometría y del álgebra.

La hipótesis principal de Piaget y Garcia en este trabajo establece que el desarrollo del conocimiento en ambos dominios está basado en los mismos instrumentos. Los instrumentos de construcción del conocimiento son las abstracciones y las generalizaciones. Asimismo, identifican los procesos resultantes de la acción de los instrumentos y los mecanismos operantes. El origen de los instrumentos se encuentra en la asimilación de objetos o hechos en estructuras de conocimiento previamente desarrolladas por los sujetos. Este proceso de asimilación incluye la acomodación, es decir, la modificación de las estructuras de conocimiento del sujeto con el fin de reequilibrarlas atendiendo a las características de los objetos o hechos.

El papel crucial desempeñado por la asimilación explica la convergencia encontrada por los autores en la compara- 
ción del desarrollo de las ideas en el individuo y en la historia (Piaget y García, 1982, p. 247):

Cada uno de nuestros capitulos ha desarrollado ejemplos tanto en la marcha de la historia como en el desarrollo mental, y la razón principal de las convergencias observadas en estos dos dominios es precisamente que el sujeto desempeña un papel activo en todo conocimiento y que la propiedad más general de sus actividades es la asimilación.

La existencia de ciertos paralelismos entre los esquemas alternativos de los alumnos y los primeros estadios del conocimiento científico en algunos campos ha sido puesta en evidencia por múltiples estudios, sobre todo en el campo de la mecánica. Algunos de ellos son citados por Matthews (1990). Estudios más recientes como los de Sequeira y Leite (1991) sobre concepciones alternativas de estudiantes portugueses en mecánica; Griffiths y Preston (1992) acerca de los errores conceptuales relacionados con las características de átomos y moléculas en estudiantes canadienses; Bar y colaboradores (1994) en relación con las ideas de niños israelíes sobre peso y caída libre; y Nardi (1994) sobre las ideas de alumnos brasileños sobre la noción de campo de fuerza, parecen confirmar las ideas defendidas por Piaget.

Por otro lado, Susan Carey, una psicóloga cognitiva, señala la necesidad de que los investigadores en la didáctica de las ciencias tengan en cuenta que filosofía e historia de la ciencia pueden hacer comprender el desarrollo conceptual de los jôvenes aprendices (Carey, 1986). Así, esta investigadora apunta las semejanzas entre la progresión de la comprensión científica en los niños y la progresión en la comprensión científica en la historia de la ciencia. En particular, toma como base el análisis de la historia de la ciencia efectuado por Kuhn (1987a y 1989), con base en paradigmas y cambios de paradigmas, para distinguir entre la reestructuración débil y fuerte en los procesos de cambio conceptual de los sujetos. Una reestructuración débil en los esquemas de conocimiento de los sujetos supone simplemente añadir nuevos conceptos y relaciones entre ellos, esto es, estamos dentro del paradigma vigente en sentido kuhninano. La reestructuración fuerte, que es equivalente a un desplazamiento de paradigma, implica un nuevo esquema de conocimiento donde los conceptos se transforman y se establecen nuevas relaciones. Esta autora, en definitiva, se centra en los paralelismos estructurales antes que en los de contenido conceptual. Nersessian (1989), por su parte, defiende que la clase de razonamiento implicado en los procesos de construcción de las representaciones científicas son los mismos en los científicos que en los estudiantes.

En relación con esto, Duschl, Hamilton y Grandy (1990) sostienen que el camino entre la psicología cognitiva y la filosofía de la ciencia tiene dos sentidos, y por ello algunos epistemólogos también han tenido en cuenta conceptos de la psicología cognitiva en sus estudios. En otros trabajos, citan el de Giere (1988) en el cual se establece la necesidad de aplicar los conceptos de la psicología cognitiva en la reestructuración de los esquemas de conocimiento para desarrollar una apropiada comprensión de los cambios de teorías científicos. En este mismo sentido, el epistemólogo e historiador de la ciencia Thomas S. Kuhn en su libro La Tensión Esencial (1987b, p. 46) apoya este mismo argumento cuando reconoce que:

Hace veinte años descubrí, y más o menos al mismo tiempo, tanto el interés intelectual por la historia de la ciencia como los estudios psicológicos de Jean Piaget. Desde entonces, ambas inquietudes han influido recíprocamente tanto en mi mente como en mi trabajo. Parte de lo que sé sobre la forma de interrogar a los científicos que ya han muerto lo aprendí examinando la forma en que Piaget interroga a los niños que estudia.

No obstante esto, no podemos dejar de citar aquí las investigaciones que ponen el acento en las limitaciones del trabajo de Piaget. Así, Deanna Kuhn (1989) mantiene que la metáfora de los niños como científicos intuitivos o ingenuos ha de interpretarse solamente con el significado de una semejanza con respecto a la comprensión científica. Esto es, ambos, niños y científicos, mejoran la comprensión del mundo mediante la construcción y revisión de una sucesión modelos mentales. Sin embargo, los procesos que guian las relaciones entre modelos mentales (teorias o paradigmas) y las nuevas evidencias, hechos $\mathrm{u}$ objetos, son significativamente diferentes. Consecuentemente, desde este punto de vista, los estudiantes no se comportan como científicos. En el razonamiento de sentido común que emplean los estudiantes no hay un verdadero control sobre la interacción modelo/evidencia, en tanto que en los procesos de desarrollo del pensamiento científico se produce una progresiva diferenciación y coordinación de teoría y evidencia. En estos procesos de desarrollo del pensamiento científico, por otra parte, se requiere reflexionar sobre las teorías, no sólo con las teorías, y sobre las evidencias, no sólo dejarse influir por ellas. En consecuencia, son procesos de desarrollo de carácter metacognitivo y estratégico. Es decir, vienen a reflejar el control sobre la interacción entre las teorías y las evidencias en el propio pensamiento.

Franco y Colinvaux-de-Dominguez (1992) critican, entre otras cosas, que Piaget no dé una explicación convincente de las diferencias entre el conocimiento científico y el conocimiento del sentido común que emplean los estudiantes. Fillon (1991), por su parte, afirma que la ontogénesis no recapitula la filogénesis porque los modelos mentales de los alumnos (modelos espontáneos) no pueden ser los de 
los científicos de épocas pasadas, ya que ni poseen los mismos conocimientos ni las mismas capacidades de razonamiento. Esto se justifica en razón de sus diferentes entornos materiales y sociales. Así, por ejemplo, los obstáculos encontrados por unos y por otros para construir conocimientos tienen origen y naturaleza diferente. Con todo, los autores apuntan que los modelos espontáneos de los alumnos tienen, en ocasiones, semejanzas sorprendentes con los modelos cientúficos de determinados momentos en la historia de la ciencia.

Por último, Duschl, Hamilton y Grandy (1990) indican que se pueden encontrar casos en que las teorías científicas en determinados momentos de la historia y las ideas de los alumnos nunca serán coincidentes. Ponen como ejemplo las teorías o modelos del Universo. Otro ejemplo lo proporciona el trabajo de Furió y Guisasola (1993), en el que se concluye que las ideas de los alumnos sobre los conceptos de carga y potencial eléctricos no pueden ser clasificadas como pertenecientes a los modelos tomados como referentes históricos.

\section{Enseñanza de la ciencia e historia de la ciencia}

Nos centraremos aquí en los beneficios didácticos que se pueden obtener con una adecuada utilización de la historia de la ciencia. En primer lugar, señalaremos que son bastantes los autores que, basándose en los paralelismos encontrados entre los modos de razonamiento espontáneo de los estudiantes y los de ciertos periodos históricos, proponen que el conocimiento por parte del profesorado de los problemas que se produjeron en el desarrollo de conceptos y teorías de las ciencias, permita a éste anticipar las dificultades que puedan tener los estudiantes en su aprendizaje de la ciencia (Wandersee, 1986; Matthews, 1994; Sequeira, 1991). Con ello, aunque no tiene ningún sentido buscar correspondencias estrictas entre las ideas de los estudiantes y las de algunos científicos a lo largo de la historia de la ciencia, ni siempre el pensamiento de los estudiantes se ha dado en alguna etapa de la evolución de la ciencia, el conocimiento de la historia de la ciencia puede poner en alerta al profesorado ante ciertas resistencias que se han manifestado a lo largo del tiempo (Saltiel, 1985).

Esta última idea en realidad tiene su origen en los estudios de Gaston Bachelard. Este epistemólogo francés introdujo en 1938, año de la primera edición de La formation de l'esprit scientifique, la noción de obstáculo epistemológico (Bachelard, 1983). Con este término, dio nombre a la resistencia que se ofreció, en determinados momentos de la historia de la ciencia, a cambios que implicasen la negación por parte de la misma ciencia de los fundamentos sobre los que se sustentaban las investigaciones anteriores. Es decir, cambios que cuestionaran alguna categoría fundamental o algún factor relativo al mismo acto de conocer. Esta resistencia, según

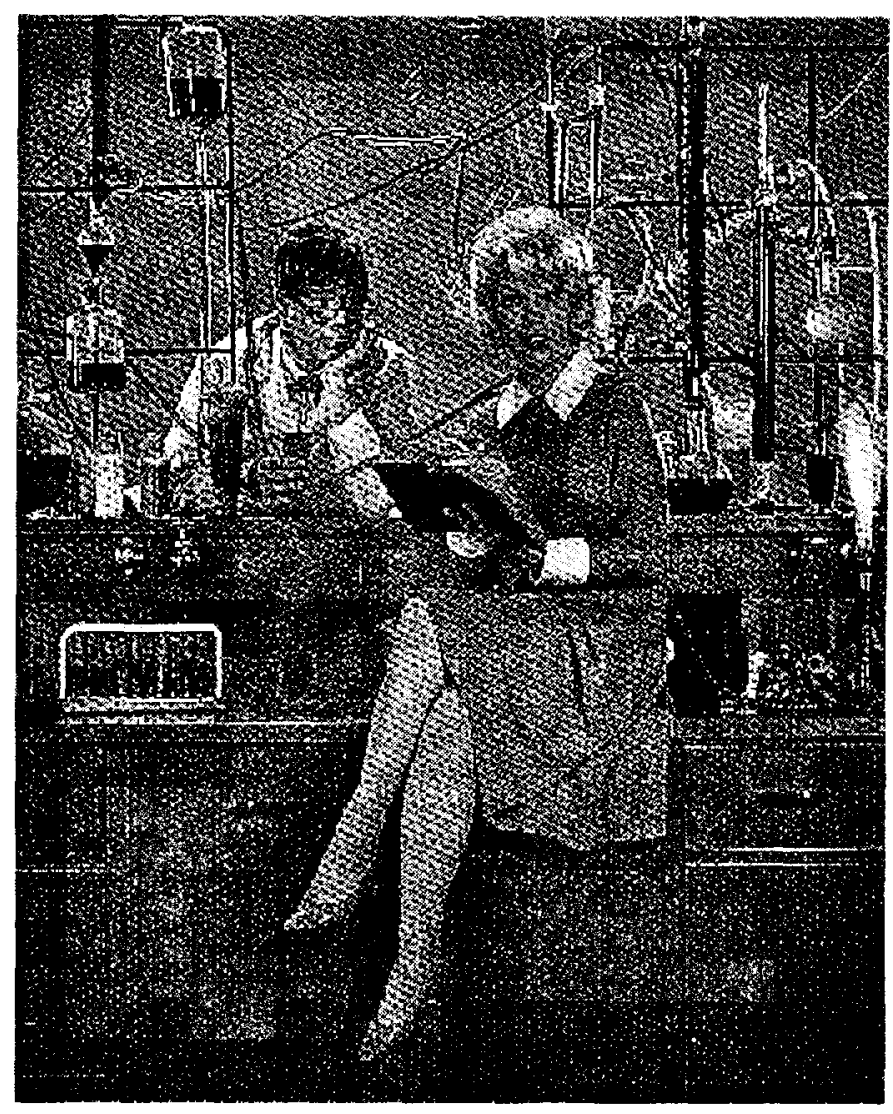

Bachelard, no se debe en exclusiva a la complejidad de lo real, esto es, a la naturaleza del objeto que nos proponemos conocer, sino a factores tales como los instintos, las costumbres intelectuales, la inercia de las culturas, la enseñanza errónea de la ciencia, la ideología de la ciencia o a los propios actos cognitivos. Esta hipótesis de Bachelard parece confirmarse posteriormente con los trabajos de A. Luria (1989), en los que se puso en evidencia el origen social de las funciones psíquicas superiores. En relación con esto, Bachelard señaló también que la noción de obstáculo épistemológico puede ser estudiada tanto en el desarrollo histórico del pensamiento científico como en los conocimientos de que dispone un estudiante de ciencias.

La historia de la ciencia en la didáctica puede ir encaminada, en segundo lugar, hacia su uso como herramienta en la enseñanza/aprendizaje (Pessoa de Calvalho, 1992; Fillon, 1991). En concreto, este último investigador hace la prometedora propuesta de efectuar actividades en las que, mediante textos históricos, los alumnos tengan posibilidad de comparar sus modelos con los de los científicos en determinados momentos de la historia. Esta estrategia, según el autor, permite centrar los objetivos en un determinado obstáculo y mostrar al aprendiz que el saber científico es el resultado de una construcción humana, con sus errores y rectificaciones 
correspondientes. De hecho, Lind (1980) ya puso de manifiesto la mayor facilidad de los estudiantes para criticar modelos aparecidos a lo largo de la historia de la ciencia que los suyos propios.

Por otra parte, la propuesta de Fillon es posible insertarla en una metodologia constructivista de enseñanza/aprendizaje de las ciencias (Driver, 1986). El profesor constructivista explora los esquemas conceptuales de los alumnos, diseña materiales y actividades que les permitan reflexionar en grupo sobre estos esquemas y mostrar sus deficiencias para, en último término, reestructurarlos hasta que lleguen a ser compatibles con los aceptados por la comunidad científica.

\section{Conclusiones}

Hemos partido de un hecho bien contrastado en la didáctica de las ciencias: nuestros alumnos tienen concepciones previas en muchos campos de las ciencias que influencian de manera decisiva su aprendizaje, y que se muestran resistentes a cualquier modificación para aproximarlas a las aceptadas por la comunidad científica.

Se ha visto también que la historia de la ciencia puede ayudarnos a comprender el desarrollo conceptual de los estudiantes, y sobre todo, ponernos en situación de entender sus dificultades y resistencias. No obstante, los procesos que ayudan a estudiantes y científicos a establecer conexiones entre los modelos mentales elaborados y las evidencias, hechos u objetos, son bien diferentes en unos y en otros. En concreto, el control y la reflexión sobre la interacción modelo/realidad, esto es, el desarrollo metacognitivo y estratégico, sólo es característico de los procesos del pensamiento científico. Además, los modelos mentales de los estudiantes no pueden coincidir exactamente con los de los científicos de épocas pasadas porque no tienen ni los mismos conocimientos ni las mismas capacidades en razón de sus diferentes entornos materiales y sociales. Incluso se han dado casos en que no hay coincidencia alguna.

Asimismo, hemos mostrado que la historia de la ciencia puede dar la posibilidad, en un contexto didáctico, de que los alumnos revisen y modifiquen sus modelos espontáneos mediante la comparación de estos modelos con los de los científicos en determinados momentos de la historia.

\section{Bibliografía}

Andersson, B. Pupils' conceptions of matter and its transformations (age 12-16), Studies in Science Education, 18, $53-85,1990$.

Ausubel, D.P., Novak, J.D. y Hanesian, H., Psicología Educativa, Trillas, México, 1986.

Bachelard, G., La formation de l'esprit scientifique. Librairie Philosophique J. Vrin, Paris, 1983.

Bar, V., Zinn, B., Goldmuntz, R. and Sneider, C., Children's concepts about weight and free fall, Science Education, 78, 149-169, 1994.

Brackenbridge, J.B., La educación de las ciencias, la historia de las ciencias y el libro de texto, las condiciones necesarias contra las suficientes, Comunicación, Lenguaje $y$ Educación, 11, 157-169, 1991.

Carey, S., Cognitive Science and Science Education, American Psychologist, 41, 1123-1130, 1986.

Chiappetta, E.L., Sethna, G.H. and Fillman, D.A., A quantitative analysis of high school chemistry textbooks for scientific literacy themes and expository learning aids, Journal of Research in Science Teaching, 28, 939-951, 1991.

Dana, T.M., The history and philosophy of science: What does it mean for science clasrooms?, The Australian Science Teachers Journal, 36, 21-26, 1990

Driver, R. \& Oldham, V., A constructivist approach to curriculum development in science, Studies in Science Education, 13, 105-122, 1986.

Driver, R., Guesne, E. y Tiberghien, A., Ideas científicas en la infancia y la adolescencia, MEC/Morata, Madrid, 1989.

Duschl R.A., Hamilton, R. and Grandy, R.E., Psychology and epistemology: match or mismatch when applied to science education, International Journal of Science Education, 12, 230-243, 1990.

Fillon, P., Histoire des sciences et réflexion épistémologique des élèves, Aster, 12, 91-120, 1991.

Franco, C. and Colinvaux-de-Dominguez, D., Genetic epistemology, history of science and science education, Science $\mathcal{E}$ Education, 1, 255-271, 1992.

Furió, C. y Guisasola, J., ¿Puede ayudar la historia de la ciencia a entender por qué los estudiantes no comprenden los conceptos de carga y potencial eléctricos?, $R \boldsymbol{e}$ vista Española de Física, 7, 46-50, 1993.

Gagné, R.M., Las condiciones del aprendizaje, Trillas, Mexico 1987.

Giere, R. Explaining Science: A cognitive approach, The University Chicago Press, Chicago 1988.

Gil, D., Contribución de la historia y de la filosofía de las ciencias al desarrollo de un modelo de enseñanza/aprendizaje como investigación, Enseñanza de las Ciencias, 11, 197-212, 1993.

Gilbert, J.K. and Zyberstajn, A., A conceptual fremework for science education: thecase study of force and movement, European Journal of Science Education, 7, 107-120, 1985.

Griffiths, A.K. \& Preston, K.R. Grade-12 Students' misconceptions relating to fundamental characteristics of atoms and molecules. Journal of Research in Science Teaching, 29, 611-628, 1992.

Hierrezuelo, J. and Montero, A., La ciencia de los alumnos. $S u$ utilización en la didáctica de la física y la química, MEC-Laia, 
Barcelona, 1989.

Kuhn, D., Children and adults as intuitive scientists, Psychological Review, 96, 674-689, 1989.

Kuhn, T.S. La estructura de las revoluciones cientificas, Fondo de Cultura Económica, Madrid, 1987a.

Kuhn, T.S., Los conceptos de causa en el desarrollo de la física, en: T.S. Kuhn, La tensión esencial, Fondo de Cultura Económica, México, 1987b, p. 46-55.

Kuhn, T.S., Qué son las revoluciones científicas y otros ensayos, Paidós/ICE-UAB, Barcelona, 1989.

Lind, G., Models in Physics: Some pedagogical reflections based on the history of science, European Journal of Science Education, 2, 15-23, 1980.

Luria, A.R., La psicología como ciencia histórica (acerca de la naturaleza histórica de los procesos psíquicos), en: Puzirei, A y Guippenréiter, Yu, El proceso de formación de la psicología marxista: Vigotski, Leontiev y Luria, Editorial Progreso, Moscú, 1989.

Llorens, J.A., De Jaime, M.C. y Llopis, R., La función del lenguaje en un enfoque constructivista del aprendizaje de las ciencias, Enseñanza de las Ciencias, 7, 111-119, 1989.

Matthews, M.R., History, philosophy and science teaching: a rapprochement, Studies in Science Education, 18, 25-51, 1990.

Matthews, M.R., Un lugar para la historia y la filosofía en la enseñanza de las.ciencias, Comunicación, lenguaje y educación,11-12, 141-155, 1991.

Matthews, M.R., Historia, filosofía y enseñanza de las ciencias: la aproximación actual, Enseñanza de las Ciencias, 12, 255-277, 1994.

Nardi, R., História da ciência $\mathrm{x}$ aprendizagem: algumas semelhanças detectadas a partir de um estudo psicognético sobre as idéias que evoluem para a noçao de campo de força, Enseñanza de las ciencias, 12, 101-106, 1994.

Nersessian, N.J., Conceptual change in science and in science education, Synthese, 80, 163-183, 1989.

Nielsen, H. and Thomsen, P.V., History and philosophy of science in physics education, International Journal of Science Education, 12, 308-316, 1990.

Osborne R. y Freyberg, P., El aprendizaje de las ciencias, Narcea, Madrid, 1991.

Pessoa de Carvalho, A.M. y Castro, R.S., La historia de la ciencia como herramienta para la enseñanza de la física en secundaria: un ejemplo en calor y temperatura, $E n$ señanza de las Ciencias, 10, 289-294, 1992.

Piaget, J., La epistemología genética, Redondo, Barcelona, 1970.

Piaget, J., Introduction a l'epistemologie genetique. P.U.F, Paris, 1974.

Piaget, J., Seis estudios de pscicología, Seix Barral, Barcelona, 1979.
Piaget,J. \& Garcia, R., Psicogénesis e historia de la ciencia, Siglo veintiuno editores, México, 1982.

Pozo, J.A., Sanz, A., Gómez Crespo, M.A. y Limón, M., Las ideas de los alumnos sobre la ciencia: una interpretación desde la psicología cognitiva, Enseñanza de las Ciencias, 9, 83-94, 1991.

Rumelhart, D.E., Schemata: the building of blocks of cognition, en R.J. Spiro, B.C. Bruce, W.F. Brewer (eds.), Theorical issues in reading comprenhension, Erlbaum, $\mathrm{Hi}$ llsdale, 1980.

Saltiel, E. y Viennot, L., ¿Qué aprendemos de las semjanzas entre las ideas históricas y el razonamiento espontáneo delos estudiantes?, Enseñanza de las Ciencias, 3, 137-144, 1985.

Schank, R.C. and Abelson, R. Plans, scripts, goals and understanding, Erlbaum, Hillsdale, 1977.

Sequeira, M. and Leite, L., Alternative conceptions and history of science in physics teacher education, Science Education, 75, 45-56, 1991.

Solaz-Portolès, J.J., Diagramas: cilustraciones eficaces en la instrucción en ciencias?, Educación Química, 7, 145-149, 1996.

Solaz-Portolés, J.J., Vidal-Abarca, E. y Sanjosé, V., Anãlisis didáctico, epistemológico e histórico de la introducción de los modelos atómicos en los textos de $2^{\circ}$ de BUP. Enseñanza de las Ciencias, número extra (IV Congreso), 283-284, 1993.

Solomon, J. Social influences on the construction of pupils' understanding of science, Studies in Science Education, 14, 63-82, 1987.

Solomon, J., Duveeen, J. and Scot, L., Teaching about the nature of science through history: Action research in the classroom, Journal of Research in Science Teaching, 29, 409-421, 1992.

Stinner, A., Science textbooks and science teaching: From logic to evidence, Science Education, 76, 1-16, 1992.

Strube, P., A content analysis of arguments and explications presented to students in physical science textbooks: a model and a example, International Journal of Science Education, 11, 195-202, 1989.

Wandersee, J.H., Can the history of science help science educators anticipate students' misconceptions?, Journal of Research in Science Teaching, 23, 581-597, 1986.

West, L.H.T. and Pines, A.L., Cognitive structure and conceptual change, Academic Press, Orlando, 1985.

\section{Agradecimientos}

Este artículo se ha enriquecido con las sugerencias formuladas por el árbitro (N. de la R.: se trata del doctor Aníbal Bascuñan Blaset), a quien le estoy muy agradecido. 